\title{
Recurrence analysis of innovation behavior of bitcoin market agents in con- ditions of COVID-19
}

\author{
Hanna Kucherova ${ }^{1, *}$, Vita Los $^{2, * *}$, Dmytro Ocheretin ${ }^{2, * * *}$, Olha Bilska $^{1, * * * *}$, and Evgenia Makazan ${ }^{2, \dagger}$ \\ ${ }^{1}$ Classic Private University, 70B Zhukovsky Str., Zaporizhzhia, 69002, Ukraine \\ ${ }^{2}$ Zaporizhzhia National University, 66 Zhukovsky Str., Zaporizhzhia, 69600, Ukraine
}

\begin{abstract}
The relevance of the research subject is explained by a fundamental change in the conditions of existence and development of agents of the digital economy, limited knowledge about their behavior under conditions of quarantine restrictions. The aim of the research is to study the series of the dynamics of the price of bitcoin and the frequency of online requests for bitcoin as an indicator of the behavior of agents of the digital economy using the methods of qualitative recurrent analysis. The types of constructed time series plots of the price of bitcoin and the frequency of requests for bitcoin are defined as drift with a superimposed linearly gradually increasing sequence, which indicates the unpredictability of the behavior of digital economy agents with a gradual stabilization in new quality trend. The scientific novelty of the research results lies in the proven connection between the series of bitcoin price dynamics and the frequency of online requests for bitcoin, tracking changes in the behavior of digital economy agents before and after the introduction of quarantine restrictions. The procedure for conducting a qualitative recurrence analysis of the series of dynamics is generalized, which takes into account the specifics of the formation of the frequency of online requests for bitcoin, the price and the behavioral aspect of its formation. The practical value lies in defining the characterization of the behavior model of digital economy agents under conditions of quarantine restrictions. The behavior of digital economy agents in the context of COVID-2019 requires further research, in particular, using cross-recurrent analysis methods.
\end{abstract}

\section{Introduction}

It is difficult to find a clear generalization of the processes that have begun and are currently taking place in the countries of the world in the COVID-pandemic $[1,2]$. Unpreparedness for new risks of this type was demonstrated by both individuals and legal entities, authorities and society as a whole. And this is expected, since the collapse of development and interaction of individual sectors of the socio-economic environment has been forming for decades. The unsystematic nature of the government's programmatic actions and the devaluation of the priority issues of ensuring the basic conditions for the survival of society and business have led to the fact that we have become hostages of our own impotence in providing the necessary resistance to the expansion of the disease and the negative socio-economic consequences of the pandemic.

New living conditions draw attention directly to the model of our behavior and in the digital market, determine that the issues of adhering to the security of interactions and the possibility of development within the new established boundaries are priority basic goals for each of us. The established models of the existence of forms and

\footnotetext{
*e-mail: kucherovahanna@gmail.com

**e-mail: vitalos.2704@gmail.com

***e-mail: odvisua@gmail.com

****e-mail: lexusol1987@ gmail.com

†e-mail: e.v.makazan@gmail.com
}

ways of life in the countries of the world have confirmed the need to reduce them, reorient the volumes of consumption, production, change business models, ways of interaction, and so on. New challenges and updated values have actualized the demand for specific goods and services that provide a solution to complex issues of socio-economic security for each in pandemic, have led to fundamental changes in the behavior of entities in all online and offline business markets. Today, timely tracking of changes in behavior in the markets contributes to the formation of a new quality of management, quick adaptation of business, changes in the basic principles of interaction and functioning of subjects of all spheres, clarification of current trends and prediction of the formation of new trends in key indicators.

An immediate rethinking of the socio-economic behavior of everyone is fundamental; the adoption of its new restrictions should take place both at the level of the subjects' consciousness and taking into account the limiting security measures. A business that has been developing offline as a priority, being partially present in the online space, while ignoring the rapid development of Internet technologies, is now unable to overcome the financial and economic losses that were incurred as a result of quarantine restrictions. Therefore, it is logical to actualize the movement of most of the business to the online space, more active use of modern digital technologies and tools. 
The parameters of consumer and business requests in the context of the pandemic have undergone fundamental changes both in structure and in quality, in the way of use. Unfortunately, modern socio-economic instruments are not able to describe the general picture of changes in demand and the behavior of economy agents, to demonstrate and reliably predict the trends of current changes in key instruments, the dynamics of which forms the profitability of the markets. Because the external environment during the pandemic has become too unpredictable. Thus, tools such as Google Trend have become useful, where the frequency of queries for keywords makes it possible to track trends in the interests of subjects of the digital market, and not only digital market in real time.

The frequency of online queries as an integrated indicator of information retrieval and the activity of economy agents determines the sphere of consumer interest, which is described by a tuple of parameters $\left\langle C_{S}, M, R_{F}, t\right\rangle$, where $C_{S}$ - semantic search characteristics (semantic core), $M$ - meaning of the semantic search, $R_{F}$ - business activity of agents (frequency of requests), $t$ - time period.

The behavioral model of economy agents is formed in such a way that with an increase in the subject's interest in the target search area, the frequency of online queries for keywords increases. If the agents' interest is not cognitive, but corresponds to the implementation of their strategy in the market, then an increase in the frequency of requests affects the price parameters of target instruments in the direction of their values increase. Thus, an upward trend in the dynamics of time series of key indicators is formed, followed by market monitoring, the results of which arouse interest in relevant modern tools.

This direct cyclical pattern of interdependence of interests in tools and the frequency of corresponding online requests requires system monitoring of their dynamics and an explanation of their changes in different periods of time, which will make it possible to determine or establish a model of agent behavior, factors influencing it and predict its development in new conditions of socio-economic system in offline and online.

Thus, the purpose of the research is the behavior of agents of the digital economy, which is described using the rate of requests for the bitcoin rate in the context of COVID-19. The object of the research is the time series: requests "bitcoin" in English and Russian of Ukrainian users of the digital market, and the price of bitcoin. The subject of research is the methods of nonlinear dynamics.

\section{Related works}

A significant number of scientific and practical works are devoted to the research of time series of cryptocurrencies, carried out by methods of machine learning and forecasting [3, 4], nonlinear autoregression [5], binomial logistic regression [6], recurrent neural network, ARIMA model [7], Bayesian neural networks [8], theory of complex systems [9-13], fractal analysis [9, 14-19] and others. However, the identification of the behavior of digital economy agents requires a search for specific parameters that would uniquely determine it. The search for such parameters is difficult, since their characterized by weak structuring and significant subjectivity. So, to date, the results of the influence of social networks on the dynamics of the indicators of the digital market [7] have been obtained, including a quantitative relationship between social networks and the bitcoin price [14-16], a connection between the parameters of bitcoin and online searches in Google has been confirmed [18], Google queries and exchange rates [18]. Based on the results of the research, the authors confirm the thesis that the indicators of online search and social networks really determine the dynamics of the rate of cryptocurrencies, therefore, this direction requires further research. Researchers $[3,7,8,20,21]$ have proved that the complexity of the processes, the peculiarities of the formation time series of bitcoin and the frequency of requests for bitcoin require the use of the methodology of nonlinear dynamics, the methods of which are actively used in the study of processes during the manifestation of crisis phenomena $[5,9]$, which in fully complies with the conditions of today. However, a fairly powerful toolkit of nonlinear dynamics only partially used in the study of the behavior of economy agents in the online information space, which proves the timeliness of this study.

\section{Matherials}

According to Google Trends data [22], the search query "bitcoin" is characterized by a simple semantic core, which serves as the basis for the formation of a more complex, refined online information search. In particular, online information requests such as "bitcoin halving", "price bitcoin", "what is bitcoin", "bitcoin course", "bitcoin dollar", "bitcoin price usd", "freebitcoin" have shown the maximum popularity in the online environment over the past year. Interest in bitcoin is not evenly distributed in Ukraine, most often in Kyiv, Kyiv region, Kharkiv, Odesa and Chernivtsi regions are interested in its price and characteristics. Google Trends the values of the frequency of requests in the Ukrainian language as insufficient in importance. Google Trends noted that the frequency of Bitcoin requests was very popular in Russian and English. At the same time, the dynamics of the frequency of requests in the Ukrainian language showed a similarity of the dynamics of the frequency of requests in Russian. The behavior of market agents in terms of the frequency of Bitcoin requests in Ukrainian the same as the frequency of Bitcoin requests in Russian and English. The dynamics of the studied time series is shown in figure 1 .

Figure 1 shows that the dynamics of indicators is characterized by a high value of volatility. The dynamic series of the frequency of requests "bitcoin" is described by a 70.58\% coefficient of variation, "bitcoin" in Russian $90.48 \%$, and the price of bitcoin $-83.43 \%$. In addition, the trend in the frequency of requests for bitcoin in English and Russian are similar, but differ in the values of the number of requests, which is quite logical, taking into account the dominant languages in the country.

The maximum value of the bitcoin price for the study period was observed on December 17, 2017 and amounted to $\$ 19140.8$, the minimum - on August 23, 


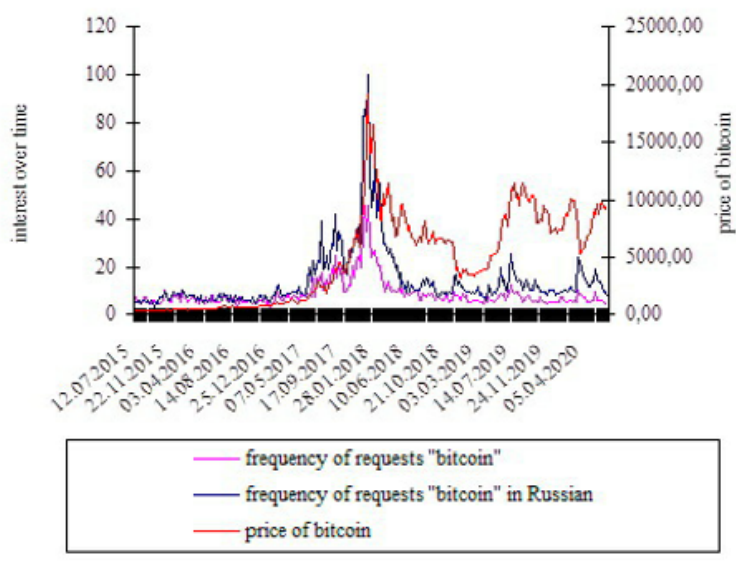

Figure 1. Frequency of requests "bitcoin", "bitcoin" in Russian on Google in Ukraine

2015 (\$228.17). However, the maximum value of the frequency of requests "bitcoin" (46 points) and "bitcoin" in Russian (100 points) was also reached on December 17, 2017. The minimum value of the series of dynamics of the frequency of requests for "bitcoin" of Russian at the level of 3 points was observed on September 27, 2015 and August 07,2016 , and the series of dynamics of the frequency of requests for "bitcoin" on December 11, 2016.

The maximum increase in the price of bitcoin [23] for the study period was recorded at the level of $41.5 \%$ (July $23,2017)$, that is, the price increased by 800.6 dollars relative to the previous period (July 16, 2017). The maximum decrease in the price of bitcoin was observed precisely at the beginning of quarantine measures in March 2020 (March 15, 2020). Bitcoin price fell by $33.5 \%$ or $\$ 2,715.8$ relative to the previous period (March 08, 2020). In general, on average, over the period under study, the price of bitcoin increased by $1.9 \%$ weekly. So, in June 2020, the price of bitcoin increased 29.4 times compared to the same period in 2015 .

Studying individual periods of the time series of dynamics, it was found that the maximum values of the price of bitcoin and the frequency of online requests for bitcoin either coincide in the period, or are very close in time. Also, sharp trends in the price of bitcoin are accompanied by a surge in interest in it, even if the price trend is decreasing. Whereas the stable fluctuations of the bitcoin price within the boundaries of a certain corridor, to a lesser extent, but still of interest to economy agents.

It is proposed to eliminate the unpredictability of the studied time series of dynamics using recurrent analysis, the implementation of which will make it possible to characterize the behavior of digital economy agents in new conditions of existence and development.

\section{Methodology}

To study the time series, the authors proposed a methodology based on the method of recurrence analysis for analyz- ing the behavior of digital economy agents in the context of COVID-19.

The input time series for the analysis were generated using data from Google Trends [22] and InvestFunds [23] for the period from July 12, 2015 to June 28, 2020. Authors investigate three time series, namely: the frequency of online requests for bitcoin in Ukraine on English and Russian, and the price of bitcoin. After the formation of the database, the behavior of the dynamics of the studied time series should be analyzed in order to establish the type of behavior and the presence of a trend or random behavior of the series.

To apply the proposed methodology, the data values of the initial series were normalized in the interval $[0,1]$ by the formula

$$
x_{n o r m}=\frac{x_{i}-x_{\min }}{x_{\max }-x_{\min }},
$$

where $x_{\text {norm }}$ - normalized time series value;

$x_{\max }, x_{\min }-$ maximum and minimum value of the time series, respectively.

After the formation of the set of initial data, the next step is the choice of the reasonable delay of the time series $(G)$. Choosing $G$ should take into account the following [18]:

- $G$ must be large enough so that the value $x(t)$ different from the value $x(t+G)$;

- but if $G$ is too large, then at time $(t+G)$ the system will lose information about what happened at time $t$.

Taking into account the above, the authors define the function of mutual information (AMI) $S$ for the time series that analyzed, taking into account nonlinear correlations [24]. The time series delay time $G$ corresponds to the first local minimum of the mutual information function (AMI). The time series reasonable delay time was calculated in the $\mathrm{R}$ environment using the tseriesChaos library.

To determine the dimension of the time series, the authors used the false nearest neighbor method given in the work [25]. This method is based on the assumption that in a correctly constructed attractor the neighboring points of the phase trajectory remain very close at the following iterations. If the nearest points diverge, then they are called false nearest neighbors. The problem is to choose a dimension of the phase space $r$ at which the fraction of points with false neighbors is minimized. The calculation of false neighbors was calculated in the R environment using the fractal library.

The next step is to build a recurrence plot based on the calculated space dimension and time delay parameters. Recurrence plot is the projection of $m$-dimensional pseudophase space onto a surface. Let the point $x_{i}$ correspond to the point of the phase trajectory $x(t)$ that describes the dynamical system in the $m$-dimensional space at the time $t=i(i=1, \ldots, N)$, then the recurrence plot is an array of points in which a nonzero element with coordinates $(i, j)$ corresponds to the case when the distance between $x_{i}$ and $x_{j}$ is less than $\gamma$ :

$$
R P_{i, j}=\theta\left(\gamma-\left\|x_{i}-x_{j}\right\|\right) x_{i}, x_{j} \in R^{m}(i, j=1, \ldots, N),
$$

where $\gamma$-the size of the neighborhood of the point $x_{i}$, 
$\left\|x_{i}-x_{j}\right\|$ - distance between points,

$\theta(\cdot)$ - Heaviside function.

The next stage of the proposed methodology is analysis of the measures of recurrence plot. This analysis makes it possible to determine the measures of complexity of the structures of recurrence plot, such as: recurrence rate, percent recurrence, percent determinism, average diagonal line length, maximum diagonal line length [16, 26, 27]. Let's consider these measures in more detail. Recurrence rate (RR) shows the density of recurrent points, that is, it characterizes the probability of repetition of system states:

$$
\mathrm{RR}=\frac{1}{N^{2}} \sum_{i, j=1}^{N} R_{i, j},
$$

where $N$ - number of points on the phase space trajectory.

Percent recurrence (\%REC) displays a decrease in the regularity of the system's behavior and is used to analyze the dynamic structure of a time series:

$$
\% R E C=\frac{\sum_{l=1}^{N} l P(l)}{N} \cdot 100 .
$$

If the percent recurrence value is less than $1 \%$, then it is said that there is a regular, clearly defined dynamics of the time series behavior. For the regularity of the system's behaviors percent recurrence value from $1 \%$ to $5 \%$. If the value of the percent recurrence lies in the range from $5 \%$ to $20 \%$ or more, then this indicates that the dynamics of the time series behavior is not regular and noisy intereventtype data. Depending on the parameters of the delay and the dimension of the phase space, the following average values of the percent recurrence can be obtained: a low ( $\%$ REC $\approx 1 \%$ to $3 \%$ ), moderate ( $\%$ REC $\approx 5 \%$ to $10 \%$ ), and high (\%REC $\approx 15 \%$ to $20 \%$ ) [28].

Percent determinism $(\% D E T)$ considers the diagonal lines of the recurrence plot. The frequency distribution of the lengths of the diagonal lines in the recurrence plot can be written as follows: $P^{\gamma}(l)=\left\{l_{i}, 1, \ldots ., N_{l}\right\}$, where $l_{i}$ - $i$-th diagonal line length, $N_{l}$ - the number of diagonal lines (each line is counted only once). If the time series is stochastic, then the diagonal lines of the recurrence diagram are very short or completely absent. And deterministic time series are characterized by long diagonals and a small number of separate recurrent points.Percent determinism $(\% D E T)$ is defined using this formula:

$$
\% D E T=\frac{\sum_{l=l_{\min }}^{N} l P(l)}{\sum_{l=l_{1}}^{N} l P(l)} \cdot 100 .
$$

Percent determinism characterizes the level of predictability of the time series.

Diagonal structures show the time during which a section of a trajectory comes close enough to another section of a trajectory. Thus, these lines allow conclusions to be drawn regarding the divergence of the trajectory elements. These indicators are average diagonal line length $(A D L)$ and maximum diagonal line length $(M D L)$.
Average diagonal line length $(A D L)$ characterizes the average time during which two sections of the trajectory pass close to each other, and can be considered as the average predictability time of the series. This measure is calculated using the formula:

$$
A D L=\frac{\sum_{l=l_{\min }}^{N} l P(l)}{\sum_{l=l_{\min }}^{N} P(l)} .
$$

Respectively, maximum diagonal line length $(M D L)$ characterizes the length of the trend and is defined as:

$$
M D L=\max \left(\left\{l_{i} ; i=1, \ldots, N_{l}\right\}\right) .
$$

Based on the analysis of the statistical characteristics of the recurrence plot, it is possible to determine the presence of homogeneous processes with independent random values, processes with slowly varying parameters, periodic or oscillating processes that correspond to nonlinear systems. Thus, the analysis of the recurrence plot allows one to evaluate the characteristics of a nonlinear object on relatively short time series, which makes it possible to make decisions regarding the object's control. The measures of the recurrence plot was calculated in the $\mathrm{R}$ environment using the nonlinearTseries library.

\section{Results}

Based on the results of the calculations, recurrence plots were obtained, the topological analysis of which makes it possible to determine the structure, type, changes in the behavior of the research object, the boundaries of phase transitions, and to establish the sensitivity of quantitative measures.

Recurrence quantification analysis (RQA) can be used not only to quantify the dynamics of a whole time-series, but also to study changes in the dynamics of a time series. Windowed RQA is potentially a very powerful tool for detecting changes in subsets of the whole time-series. To conduct a better study of the temporal structure, the dynamic series of the bitcoin request frequency (in English) and time-series of price of bitcoin were divided into four periods, the length of each of which was 65 observations: A) July 12, 2015 - October 02, 2016, B) October 09, 2016 - December 31, 2017, C) January 07, 2018 - March 31, 2019, D) April 07,2019 - June 28, 2020. Recurrence plot of the behavior of digital economy agents were constructed and RQA was calculated for each subset of the time series (adjacent non-overlapping windows of 65 data points) (figure 2).

Gradual changes in the parameters of the behavior of digital economy agents are clearly visible, in particular during period B (October 09, 2016 - December 31, 2017), where the drift of the attractor was revealed (white lower and upper corners of the plot, a diagonal line) and the emergence of a new structure during the period of influence of the consequences COVID-2019 in period D (April 07,2019 - June 28, 2020). 


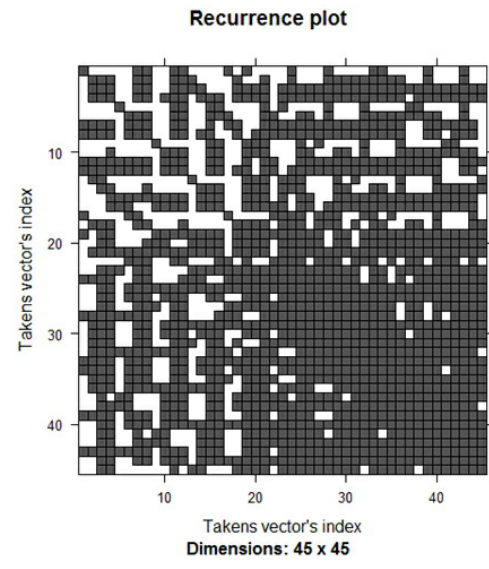

A)

Recurrence plot

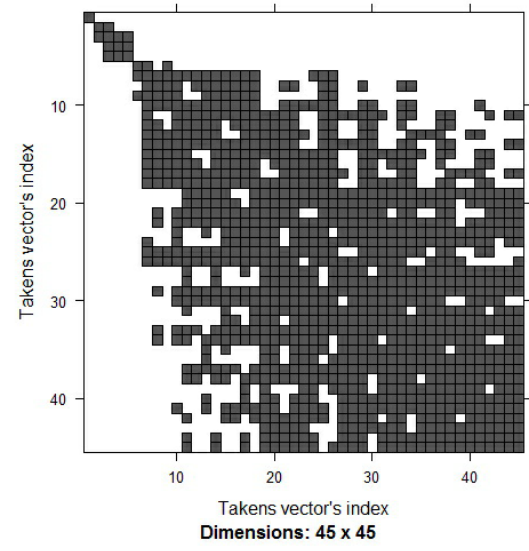

C)

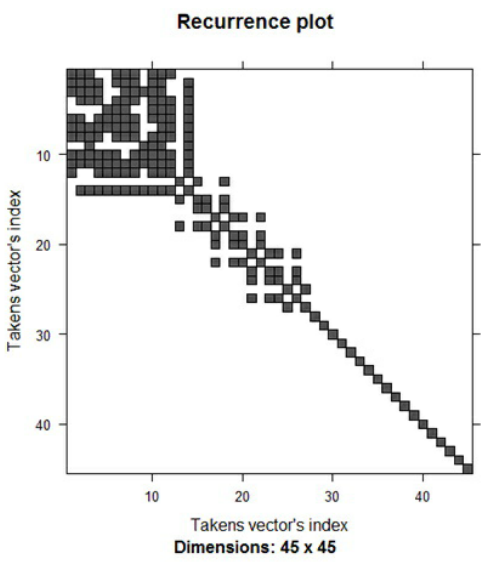

B)

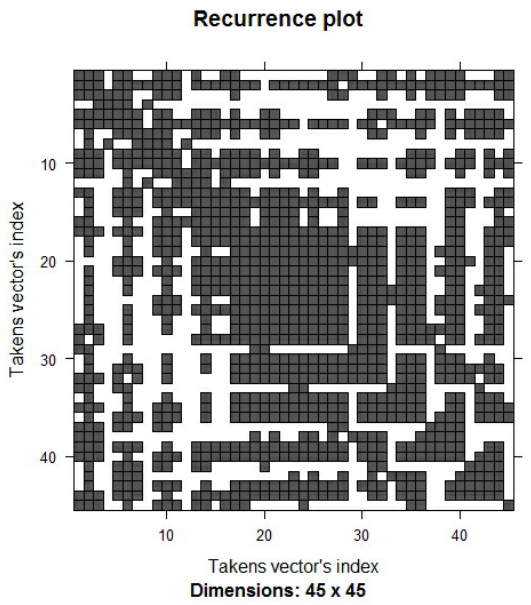

D)

Figure 2. Recurrence plot of the behavior of digital economy agents (frequency of online requests "bitcoin" according to Google Trends in Ukraine): A) July 12, 2015 - October 02, 2016, B) October 09, 2016 - December 31, 2017, C) January 07, 2018 - March 31, 2019, D) April 07,2019 - June 28, 2020

Analyzed period on the recurrence plot is displayed by contrasting areas and stripes, which is explained by sharp changes in values, randomness, rarity, in general, stochastic behavior of digital market entities, unpredictability of changes in interest in it.

However, separately placed random recurrent points are not the result of randomness, their presence proves that interest in the price of bitcoin is simply not stable over time and can be caused by significant fluctuations. The measures of the recurrence plot are investigated such as: percent recurrence (\%REC), percent determinism (\%DET), average diagonal line length $(A D L)$, maximum diagonal line length $(M D L)$ (table 1).

The change in the \%REC value from $71.46 \%$ to $57.93 \%$ in the periods under study proves that the behavior of digital market entities is unstable, noisy interevent-type data. The lowest value of \%REC $(9.83 \%)$ was recorded in period $\mathrm{B}$, when the trajectory of behavior reaches a new level of development of processes. Starting from period $\mathrm{C}$, the \%REC value increases significantly and fluctuates within the range of $54-57 \%$. The obtained values indicate an increase in irregularity and the presence of noisy
Table 1. Recurrence Quantification Analysis of the frequency of requests "bitcoin"

\begin{tabular}{lllll}
\hline $\begin{array}{l}\text { Measures of the } \\
\text { recurrence plot }\end{array}$ & $\begin{array}{l}\text { 1 plot } \\
\text { (A) }\end{array}$ & $\begin{array}{l}2 \text { plot } \\
\text { (B) }\end{array}$ & $\begin{array}{l}\text { 3 plot } \\
\text { (C) }\end{array}$ & $\begin{array}{l}4 \text { plot } \\
\text { (D) }\end{array}$ \\
\hline \%REC & 71.46 & 9.83 & 54.67 & 57.93 \\
\%DET & 93.78 & 73.87 & 93.86 & 89.60 \\
ADL & 4.80 & 3.97 & 5.74 & 3.12 \\
MDL & 35.00 & 6.00 & 39.00 & 27.00 \\
\hline
\end{tabular}

interevent-type data in this time series. Percent determinism (\%DET) ranges from $73-93 \%$. In addition, in periods $\mathrm{A}$ and $\mathrm{C}$ the measure value is almost the same, while in period D it slightly decreased to $89.60 \%$.

Average trend predictability time $(A D L)$ and maximum diagonal line length $(M D L)$ are lowest in periods $\mathrm{B}$ and $\mathrm{D}$. Want in period $\mathrm{D}$ the $M D L$ value reaches 27 points, which is 4.5 times more than the same value in period B. Thus, the trajectory of the behavior of digital economy agents demonstrates a phase transition to another level of development in period $\mathrm{B}$ and the formation of a 
new, approximate model to the existing one, but with new qualitative parameters in period $\mathrm{D}$.

At the same time, the recurrence plots for the corresponding periods of the bitcoin price are also characterized by the attractor drift. However, the dynamics of the indicator adheres to the original trajectory from September 10, 2016 and takes into account the changes in processes during the $\mathrm{D}$ period as a result of the impact of COVID-2019 (figure 3). Despite the powerful volatility and extreme growth in the price of bitcoin (in particular in 2018), the symmetry of plots proves the constancy of the formation of bitcoin price over time, the tendency of points to a given trajectory, but with the inherent randomness of qualitative values. The measures of the recurrence plot for price of bitcoin are in table 2 .

Table 2. Recurrence Quantification Analysis of the price of bitcoin

\begin{tabular}{lllll}
\hline $\begin{array}{l}\text { Measures of the } \\
\text { recurrence plot }\end{array}$ & $\begin{array}{l}\text { 1 plot } \\
(\mathrm{A})\end{array}$ & $\begin{array}{l}\text { 2 plot } \\
(\mathrm{B})\end{array}$ & $\begin{array}{l}3 \text { plot } \\
\text { (C) }\end{array}$ & $\begin{array}{l}4 \text { plot } \\
\text { (D) }\end{array}$ \\
\hline \%REC & 1 & 37.5 & 24.5 & 17.5 \\
$\%$ DET & 99.5 & 82.67 & 77.55 & 82.86 \\
ADL & 19 & 12 & 10 & 5 \\
MDL & 10.76 & 6.53 & 4.47 & 3.87 \\
\hline
\end{tabular}

The \%REC value of the series of bitcoin price dynamics is lower than the value of the similar measure of the frequency of requests for bitcoin, which proves the more constant behavior of digital economy agents. However, in period $\mathrm{A}$, a time series of bitcoin price dynamics were characterized by regularity and clear definition of the dynamics of changes in the time series $(\%$ REC $=1 \%)$.

The indicators of predictability of the time series (\%DET) are similar with the dynamics of changes in requests for bitcoin in periods $\mathrm{B}$ and $\mathrm{D}$, but differ significantly in values. In particular, the lowest values of the measure were recorded in period $\mathrm{C}$.

However, the time series of bitcoin price dynamics are characterized by a high level of unpredictability, the average predictability time significantly decreased from 19 points in period A to 5 points in period D. The length of the trend decreased from 10.76 points in period A to 3.87 points in period D. Another result was obtained from the data of recurrence plot of agents of the digital economy for the indicator of the frequency of requests "bitcoin" in Russian (figure 4, figure 5). And this is logical, since Ukraine is a bilingual country (Ukrainian and Russian languages).

In figure 5, period A clearly shows the drift of the attractor, the formation of a strong trend in the price of bitcoin, which is displayed in the form of an arrow. The formation of a trend is accompanied by an increase in interest in price changes, the frequency of requests is not stable in time for the frequency and value (figure 4, A).

Black stripes characterize nonstationarity of behavior, which means the formation of a transitional period. Periodic patterns characterize the cyclical nature of certain changes in interest in the price of bitcoin, the distance between which determines the period. Black dots, which are repeated in isolation, characterize a random interest in the price of bitcoin, its rapid changes (figure 4, A). The formation of the price trend, which is presented on the recurrence plot for period B (figure 5, B), is characterized by a certain stationarity of fluctuations within the accepted range of values. The fading of bitcoin price fluctuations is accompanied by the systematization of interest in it, streamlining the behavior of digital economy agents, reducing it to the usual monitoring of prices (figure 4, B). The measures of the recurrence plot for price of bitcoin and frequency of online requests "bitcoin" in Russian are in table 3. The constancy and regularity of the bitcoin price dynamics significantly increases in period B compared to period $\mathrm{A}$, the $\%$ REC value changed from $54.71 \%$ to $5.41 \%$. However, the percent determinism (\%DET) is reduced from $97.97 \%$ to $82.61 \%$ in the corresponding periods. Over the selected periods, the average predictability time decreased from 19.08 points to 5.7 points, and the trend length decreased by half - from 77 points to 35 points. There is a change in the phase space, trends in general. Other trends show metrics for the time series of bitcoin requests. The percent determinism (\%DET) is decreasing, from $97.97 \%$ to $82.61 \%$ in the corresponding periods. The average predictability level slightly decreased from 4 points to 3.9 points, and the trend length increased from 13 points to 19 points, which confirms the formation of a clear trend and a strong trend.

\section{Conclusions}

As a result of the research, the relationship between the time series of the price of bitcoin and the frequency of online requests for bitcoin as an indicator that characterizes the behavior of agents of the digital economy. The behavior differs were confirmed in the periods before and after the introduction of the COVID-19 quarantine restrictions. An increase in the activity and internal disturbances in the behavior of agents of the digital economy during periods of significant volatility in the price of bitcoin and, on the contrary, its decrease during a period of stabilization and insignificant fluctuations in a certain price band was confirmed. This pattern is explained by the decentralization of the digital market tools, the complexity of the processes of internal self-organization of agents, the strategies they have chosen in the digital market, their role, emotionalvolitional, behavioral and cognitive features that are uncommonly manifested during quarantine restrictions. The approach and procedure for the implementation of the Recurrence Quantification Analysis (RQA) of the time series of the price of bitcoin and the frequency of online requests about it are determined. The investigated recurrence plots of the bitcoin price and the frequency of online requests for bitcoin indicate the unpredictability of the behavior of digital economy agents with a pronounced linear trend. As a result of the research of windowed RQA, the authors came to the conclusion that the behavior of the agents of the digital economy (the frequency of requests for "bitcoin" in English) showed a change in trend precisely in the period October 09,2016 - March 31, 2019, where the drift of the attractor and the emergence of a new structure were revealed. During the period of COVID-2019 quarantine re- 


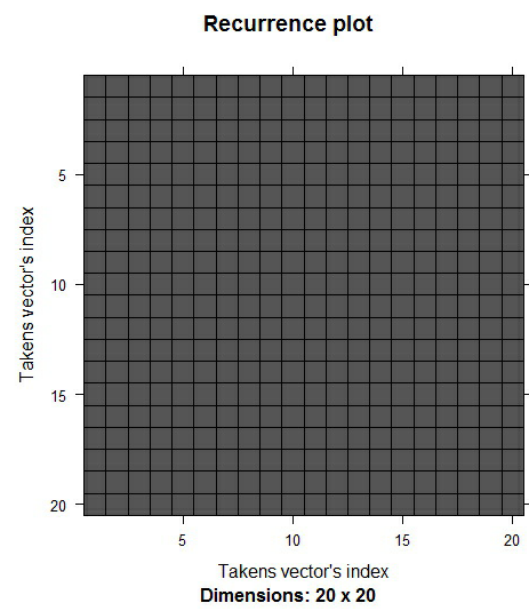

A)

Recurrence plot

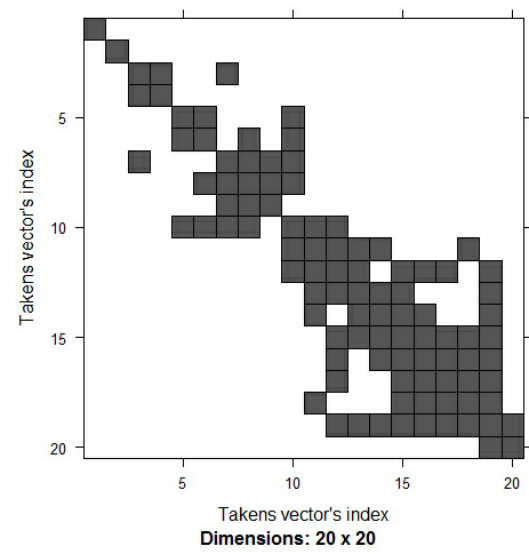

C)

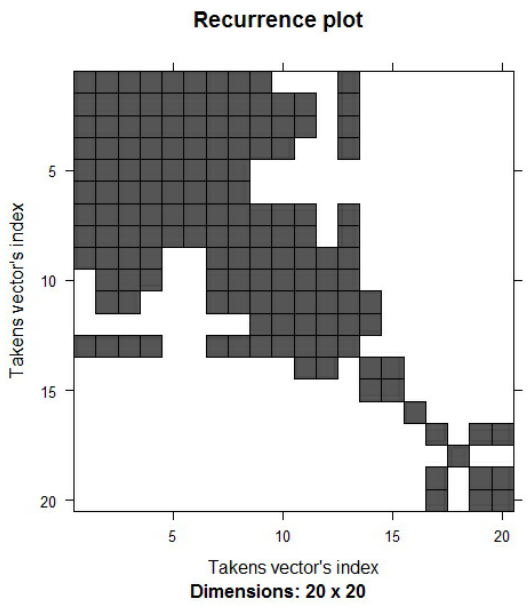

B)

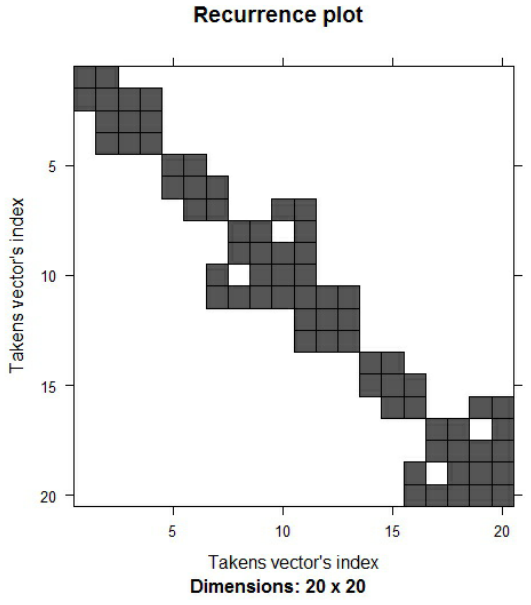

D)

Figure 3. Recurrence plot of the price of bitcoin: A) July 12, 2015 - October 02, 2016, B) October 09, 2016 - December 31, 2017, C) January 07, 2018 - March 31, 2019, D) April 07,2019 - June 28, 2020

Table 3. Recurrence Quantification Analysis of the price of bitcoin and frequency of online requests "bitcoin" in Russian

\begin{tabular}{lllll}
\hline \multirow{2}{*}{ Measures of the recurrence plot } & \multicolumn{2}{l}{ Price of bitcoin } & \multicolumn{2}{l}{ Frequency of online requests "bitcoin" in Russian } \\
\cline { 2 - 5 } & 1 plot (A) & 2 plot $(\mathrm{B})$ & 1 plot $(\mathrm{A})$ & 2 plot $(\mathrm{B})$ \\
\hline \%REC & 54.7 & 5.41 & 20.07 & 39.45 \\
\%DET & 98 & 82.6 & 71.19 & 74.71 \\
ADL & 19.1 & 5.7 & 4 & 3,9 \\
MDL & 77 & 35 & 13 & 19 \\
\hline
\end{tabular}

strictions, the behavior of economy agents is characterized by an increased level of stochastic, although interest in the price of bitcoin is unstable over time, but can be caused by significant fluctuations. A qualitative analysis of recurrence plots of the behavior of digital economy agents confirmed the formation of a new phase transition and destabilization during the period of quarantine restrictions, which forms a behavior model similar to the previous one, but with new qualitative parameters. The bitcoin price is characterized by the drift of the attractor, the constancy of price formation over time, adherence to the initial trajectory and the tendency of points towards it with a characteristic randomness of qualitative values, which is explained by changes in socio-economic processes, in particular as a result of the impact of COVID-2019. However, the time series of the bitcoin price and the frequency of requests "bitcoin" in Russian are qualitatively different from the similar series of the frequency of requests "bitcoin" in English for Ukrainian online-user. The investigated series of bitcoin price dynamics over two periods also demonstrates the drift of the attractor and the formation of a strong trend, which is accompanied by an increase and stabilization of interest in price fluctuations. The frequency of online requests is unstable over time only during the period July 12, 2015 - December 31, 2017. The non-stationarity of the behavior of economy agents was confirmed, the formation of a transitional period with a characteristic alternation of cyclical and random changes in interest in the 


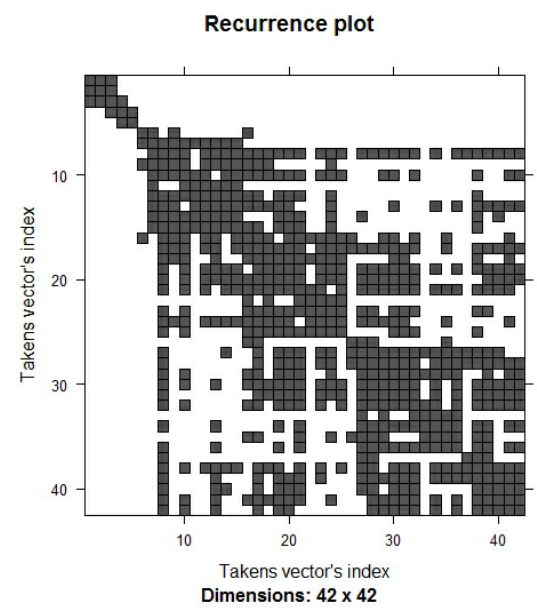

A)

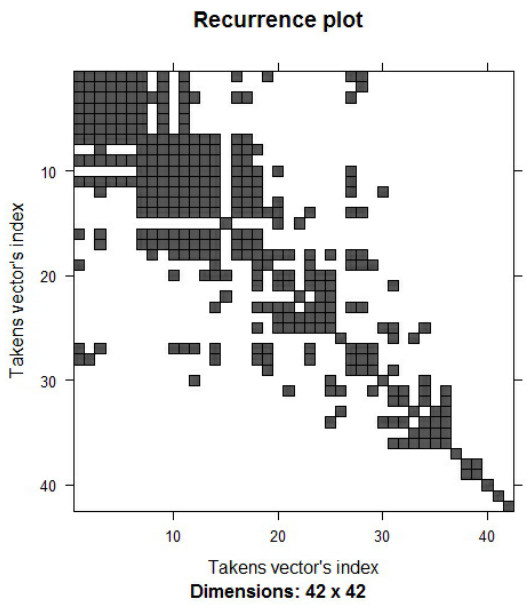

B)

Figure 4. Recurrence plot of the behavior of digital economy agents (frequency of online requests "bitcoin" in Russian according to Google Trends in Ukraine): A) July 12, 2015 - December 31, 2017, B) January 07, 2018 - June 28, 2020

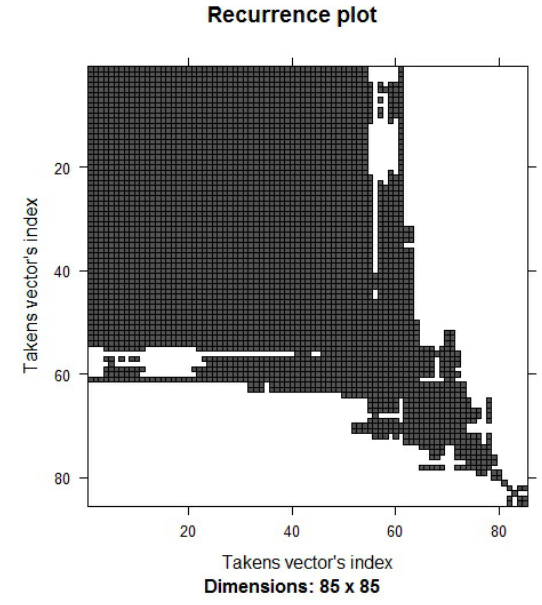

A)

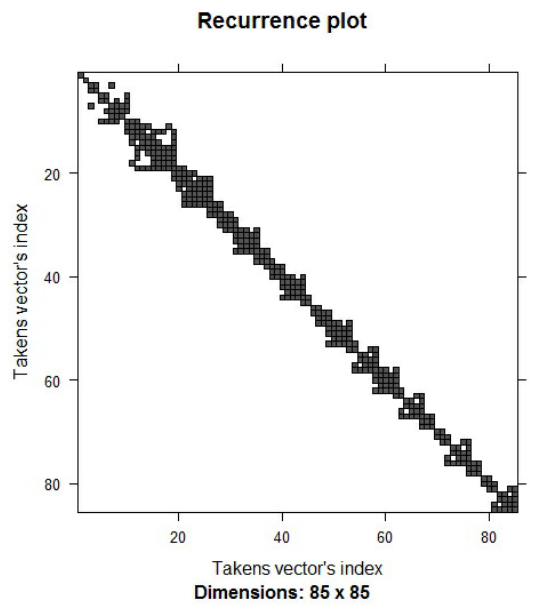

B)

Figure 5. Recurrence plot of the price of bitcoin: A) July 12, 2015 - December 31, 2017, B) January 07, 2018 - June 28, 2020

bitcoin price in the period July 12, 2015 - December 31, 2017 was recorded. Whereas the period January 07, 2018 - June 28, 2020 is characterized by a certain stationarity of fluctuations, which is accompanied by the systematization of interest in the price of bitcoin, streamlining the behavior of agents of the digital economy, reducing it to the usual monitoring of prices. Thus, the behavior of digital economy agents in the context of COVID-2019 has generated a new trend with updated qualitative indicators, which requires further research, including using cross-recurrent analysis methods.

\section{References}

[1] S. Semerikov, S. Chukharev, S. Sakhno, A. Striuk, V. Osadchyi, V. Solovieva, T. Vakaliuk, P. Nechypurenko, O. Bondarenko, H. Danylchuk, E3S Web of Conferences 166 (2020)

[2] S. Semerikov, H. Kucherova, V. Los, D. Ocheretin, CEUR Workshop Proceedings 2845, 22 (2021)
[3] P.M. Krafft, N. Della Penna, A.S. Pentland, p. 1-13 (2018)

[4] M. Saad, J. Choi, D. Nyang, J. Kim, A. Mohaisen, IEEE Systems Journal 14, 321 (2020)

[5] N. Indera, I. Yassin, A. Zabidi, Z. Rizman, Journal of Fundamental and Applied Sciences 9, 791 (2017)

[6] I. Madan, S. Saluja, A. Zhao, Automated bitcoin trading via machine learning algorithms (2014), https://tinyurl.com/jb5r93w

[7] S. McNally, Master's thesis, Dublin, National College of Ireland (2016), http: //norma.ncirl.ie/ id/eprint/2496

[8] H. Jang, J. Lee, IEEE Access 6, 5427 (2017)

[9] V. Soloviev, A. Belinskiy, Communications in Computer and Information Science 1007, 276 (2019)

[10] A. Bielinskyi, V. Soloviev, CEUR Workshop Proceedings 2292, 37 (2018)

[11] V. Soloviev, A. Belinskij, CEUR Workshop Proceedings 2104, 116 (2018) 
[12] V. Soloviev, S. Yevtushenko, V. Batareyev, CEUR Workshop Proceedings 2546, 87 (2019)

[13] V. Soloviev, V. Solovieva, A. Tuliakova, A. Hostryk, L. Pichl, CEUR Workshop Proceedings 2713, 53 (2020)

[14] L. Kristoufek, Scientific Reports 3, 3415 (2013)

[15] D. Garcia, C.J. Tessone, P. Mavrodiev, N. Perony, Journal of The Royal Society Interface 11, 20140623 (2014)

[16] L. Kirichenko, T. Radivilova, V. Bullakh, V. Chakryan, International Journal Information Technologies and Knowledge 12.1, 43 (2018)

[17] H. Kucherova, Actual Problems of Economics 8, 122 (2019)

[18] H. Kucherova, D. Ocheretin, V. Los, O. Bilska, Recurrence Analysis of the Economic Behavior of Agents of the Frequency of Online Exchange Rate in the Information Environment, in Proceedings of the III International Scientific Congress Society of Ambient Intelligence 2020 (ISC-SAI 2020) (Atlantis Press, 2020), pp. 279-288, ISBN 978-94-6252-9335, ISSN 2352-5428, https://doi .org/10.2991/ aebmr.k.200318.035

[19] H. Danylchuk, O. Kovtun, L. Kibalnyk, O. Sysoiev, E3S Web of Conferences 166 (2020)
[20] Z.H. Munim, M.H. Shakil, I. Alon, J. Risk Financial Manag. 12, 103 (2019)

[21] G.C. Dumitrescu, Global Economic Observer 5, 63 (2017)

[22] Google Trends Home page (2021), https:// trends.google.com . ua/trends/?geo=UA

[23] Bitcoin. InvestFunds. Home page (2021), https:// invest funds.ru/indexes/9021

[24] R. Hegger, H. Kantz, T. Schreiber, Chaos: An Interdisciplinary Journal of Nonlinear Science 9, 413 (1999)

[25] M.B. Kennel, R. Brown, H.D.I. Abarbanel, Phys Rev A 45, 3403-3411 (1992)

[26] A. Fabretti, M. Ausloos, Int J Mod Phys C 16, 671 (2005)

[27] F. Birleanu, C. Ioana, C. Gervaise, J. Chanussot, A. Serbanescu, G. Serban, On the recurrence plot analysis method behaviour under scaling transform, in 2011 IEEE Statistical Signal Processing Workshop (SSP) (IEEE, Nice, France, 2011), pp. 789-792, https://ieeexplore.ieee.org/ document $/ 5967823$

[28] S. Wallot, Discourse Process 54, 382 (2017) 